\title{
Lorentz Transformation in Super System and in Super System of Photon
}

\author{
Mukul Chandra Das ${ }^{1, *}$, Rampada Misra ${ }^{2}$ \\ ${ }^{1}$ Satmile High School, Satmile - 721452, Contai, West Bengal, India \\ ${ }^{2}$ Department of Physics (P.G.), Midnapore College, Midnapore - 721102, West Bengal, India \\ *E-mail address: mukuldas.100@gmail.com
}

\begin{abstract}
Lorentz transformation considers that relative velocity of the frame of references in inertial system is less than the velocity of light. If it be such that a frame is moving with velocity same as that of light with respect to a frame of observer then, Lorentz transformation in it will not be same as done in inertial system. Again photon is not only a particle or wave but it is a complex system due to the fact that it possesses spin and linear motion simultaneously. So, it will have some complex characteristics. In this work first, trial would be made to find out the process of Lorentz transformation in between two frames having relative velocity same as that of light and then, this concept, would be applied in the system of photon.
\end{abstract}

Keywords: Lorentz transformation; Electromagnetic field; gravitational field; photon

\section{INTRODUCTION}

It is well known that if a body or particle having rest mass $m_{0}$ moves with rectilinear velocity $v$ with respect to an observer in an inertial system then its relativistic mass [1] is

$$
m=m_{0} / \sqrt{1-v^{2} / c^{2}}=\gamma m_{0}
$$

Similarly, if a body having radius of gyration $a$ about an axis, moves with angular velocity $\omega$ with respect to an observer then its relativistic mass [2] would be

$$
m=m_{0} / \sqrt{1-\omega^{2} a^{2} / c^{2}}
$$

It is well known that if an inertial frame moves with uniform velocity along the $\mathrm{X}$ axis with respect to an observer, then Lorentz transformation between these two frames are as shown below 


$$
\left(\begin{array}{l}
x^{\prime} \\
y^{\prime} \\
z^{\prime} \\
t^{\prime}
\end{array}\right)=\left(\begin{array}{cccc}
\gamma & 0 & 0 & -\gamma v \\
0 & 1 & 0 & 0 \\
0 & 0 & 1 & 0 \\
-\frac{\gamma v}{c^{2}} & 0 & 0 & \gamma
\end{array}\right)\left(\begin{array}{l}
x \\
y \\
z \\
t
\end{array}\right) \quad, \quad\left(\begin{array}{l}
x \\
y \\
z \\
t
\end{array}\right)=\left(\begin{array}{cccc}
\gamma & 0 & 0 & \gamma v \\
0 & 1 & 0 & 0 \\
0 & 0 & 1 & 0 \\
\frac{\gamma v}{c^{2}} & 0 & 0 & \gamma
\end{array}\right)\left(\begin{array}{l}
x^{\prime} \\
y^{\prime} \\
z^{\prime} \\
t^{\prime}
\end{array}\right)
$$

Now, if one considers that a frame is moving with velocity same as that of light along $\mathrm{X}$ axis with respect to an observer then, relations for transformation of co-ordinates from moving frame to observer's frame will not be exactly same as shown in (3) due to the fact that $\gamma$ will be infinite and the system may be called super system.. In the present work, trial has been made to frame a super system.

According to the discussion in [3] photon is not only a particle or a wave but it is a system and a kind of spin-linear (SL) interaction is happening in this system. The system is denoted by the term 'Super System of Photon' (SSP). This system has been framed by considering four inertial frames in a superimposed form [3]. Again, photon contains electromagnetic field $\psi_{\alpha}(r, t)$ accompanying the gravitational field $G_{\alpha}^{\prime}\left(r^{\prime}, t^{\prime}\right)$. Relation between these two fields in SSP is

$$
G_{\alpha}^{\prime}\left(r^{\prime}, t^{\prime}\right)=\chi Z_{i j} \psi_{\alpha}(r, t), \quad \psi_{\alpha}(r, t)=\bar{\chi} \bar{Z}_{i j} G_{\alpha}^{\prime}\left(r^{\prime}, t^{\prime}\right)
$$

where, $Z_{i j}$ and $\bar{Z}_{i j}$ are coordinate transformation matrices in SSP as discussed in [3] also $\chi$ and $\bar{\chi}$ are two constant. But, SSP must not be the composition of inertial frame of references rather, it is the composition of super systems due to the fact that photon may be considered as a small mass concentrated in a ring of radius $r$ and rotates at velocity of light and it also has linear motion with velocity of light along the axis of rotation.

So, in this work a trial has been made to correct the frame of SSP to re-write the correct form of equation (4)

\section{ENERGY - VELOCITY RELATION IN SUPERSYSTEM}

Let a body of mass $m_{0}$ starts its journey from rest and at any instant its velocity and kinetic energy respectively are $v$ and $E_{k}$. Also let, this kinetic energy is not supplied from external source rather; it is supplied from internal source reducing mass $(\Delta m)$ from rest mass of the body. This means that kinetic energy attributed to the body $E_{k}=\Delta m c^{2}$ where, $\Delta m$ come from rest mass $m_{0}$. So, in every instant total relativistic mass of the body will remain unchanged and this will be $m_{0}$. If, $v$ is increased (or kinetic energy is increased) then $\Delta m$ will be increased, therefore, mass excluding kinetic energy of the moving body will be $\left(m_{0}-\Delta m\right)$. So, at any instant, one can write 


$$
m_{0}=\frac{m_{0}-\Delta m}{\sqrt{1-v^{2} / c^{2}}}
$$

Again, we obtain from (5) the total relativistic mass at any instant would be

$$
m_{0}=\frac{\Delta m}{1-\sqrt{1-v^{2} / c^{2}}}=k \Delta m
$$

where, $k=1 / 1-\sqrt{1-v^{2} / c^{2}}$. Therefore, one can write from (6), total energy of the system

$$
m_{0} c^{2}=k \Delta m c^{2}
$$

It is clear that $\Delta m c^{2}$ is kinetic energy attributed to the body where, $\Delta m$ has been reduced from rest mass of the body and total energy $\left(m_{0} c^{2}\right)$ of the body always remain unchanged. Now, equations (6) and (7) imply that if, $\Delta m \rightarrow m_{0}$ then $v \rightarrow c$ and $k \rightarrow 1$.

This means that if a particle possesses energy totally kinetic (i.e. $\Delta m c^{2}=m_{0} c^{2}$ ) then its velocity will be $c$ (velocity of light) and this condition leads to the result that $k=1$. In this circumstance comparing (1) and (6) one can write $k(=1)$ which is analogous or equivalent to $\gamma$. Now the system in which the particle is attributed is known as Super System.

\section{LORENTZ TRANSFORMATION IN SUPER SYSTEMS}

If $S$ and $S^{\prime}$ are two frames present in a super system, $S$ is the observer's frame and $S^{\prime}$ is moving with uniform velocity $c$ (velocity of light) along positive direction of $X$ axis with respect to $S$. Then Lorentz transformation would be like (3) but there $\gamma$ would be $k(=1)$ and $v=c$. Therefore using $k(=1)$ instead of $\gamma$ and $c$ instead of $v$ we obtain from (3) the Lorentz transformations in Super System as shown below

$$
\left(\begin{array}{l}
x^{\prime} \\
y^{\prime} \\
z^{\prime} \\
t^{\prime}
\end{array}\right)=\left(\begin{array}{cccc}
1 & 0 & 0 & -c \\
0 & 1 & 0 & 0 \\
0 & 0 & 1 & 0 \\
-1 / c & 0 & 0 & 1
\end{array}\right)\left(\begin{array}{l}
x \\
y \\
z \\
t
\end{array}\right) \quad\left(\begin{array}{l}
x \\
y \\
z \\
t
\end{array}\right)=\left(\begin{array}{cccc}
1 & 0 & 0 & c \\
0 & 1 & 0 & 0 \\
0 & 0 & 1 & 0 \\
1 / c & 0 & 0 & 1
\end{array}\right)\left(\begin{array}{l}
x^{\prime} \\
y^{\prime} \\
z^{\prime} \\
t^{\prime}
\end{array}\right)
$$

Now, relative velocity in between two frames $S$ and $S^{\prime}$ is $c$ along $X$ axis. If, at any instant, $X$ coordinate be $x$ and time coordinate be $t$ then, $x=c t$. Therefore,

$$
x^{2}-c^{2} t^{2}=0
$$

Similarly one can write $x^{\prime 2}-c t^{2}=0$. However, using (8) and (9) we obtain

$$
x^{\prime 2}+y^{\prime 2}+z^{\prime 2}-c^{2} t^{\prime 2}=(x-c t)^{2}+y^{2}+z^{2}-c^{2}(t-x / c)^{2}=x^{2}+y^{2}+z^{2}-c^{2} t^{2}
$$


which is the essential condition for the transformation of coordinate from one frame of reference to another frame of reference.

\section{CO-ORDINATE TRANSFORMATION IN SUPER SYSTEM OF PHOTON (SSP)}

Photon has spin angular momentum about an axis or along the direction of propagation. Photon may be considered as a small mass concentrated in a ring of radius $r$ and rotates at velocity of light $(u=c)$ and it also has linear motion with velocity of light along the axis of rotation $[3,4]$. This means that photon is the composition of three reference frames $\left(S_{1}, S_{2}\right.$, and $S_{3}$ ) with respect to observer's frame $(S)$ and the system is known as Super System of Photon (SSP) [3].

In the picture of SSP, frames $S$ and $S_{1}$ have both their $X$ axes aligned and $S_{1}$ is rotating at an angular velocity $\omega$ about $X_{1}$ axis as observed by $S$. The frame $S_{1}$ has another coordinate reference frame $S_{2}$, where $X_{2}$ axis of $S_{2}$, are rotated by an angle $\theta$ counter clockwise with respect to $S_{1}$ on $X_{1} Y_{1}$ plane. Frames $S_{2}$ and $S_{3}$ have both their co-ordinate axes aligned and $S_{3}$ is moving at a linear velocity $c$ (velocity of light) along $\mathrm{X}_{3}$ axis as observed by $S_{2}$ then coordinate transformation matrix from $S$ to $S_{3}$ would be $A_{i j}$

$$
A_{i j}=\left(\begin{array}{cccc}
1 & 0 & 0 & -c \\
0 & 1 & 0 & 0 \\
0 & 0 & 1 & 0 \\
-1 / c & 0 & 0 & 1
\end{array}\right)\left(\begin{array}{cccc}
\cos \theta & \sin \theta & 0 & 0 \\
-\sin \theta & \cos \theta & 0 & 0 \\
0 & 0 & 1 & 0 \\
0 & 0 & 0 & 1
\end{array}\right)\left(\begin{array}{cccc}
1 & 0 & 0 & 0 \\
0 & \cos \omega t & \sin \omega t & 0 \\
0 & -\sin \omega t & \cos \omega t & 0 \\
0 & 0 & 0 & 1
\end{array}\right)
$$

Similarly the matrix for coordinate transformation from $S_{3}$ to $S$ would be

$$
B_{i j}=\left(\begin{array}{cccc}
1 & 0 & 0 & 0 \\
0 & \cos \omega t & -\sin \omega t & 0 \\
0 & \sin \omega t & \cos \omega t & 0 \\
0 & 0 & 0 & 1
\end{array}\right)\left(\begin{array}{cccc}
\cos \theta & -\sin \theta & 0 & 0 \\
\sin \theta & \cos \theta & 0 & 0 \\
0 & 0 & 1 & 0 \\
0 & 0 & 0 & 1
\end{array}\right)\left(\begin{array}{cccc}
1 & 0 & 0 & c \\
0 & 1 & 0 & 0 \\
0 & 0 & 1 & 0 \\
1 / c & 0 & 0 & 1
\end{array}\right)
$$

These leads to the relation for coordinate transformation from $S_{3}$ to $S$ and from $S$ to $S_{3}$ respectively

$$
\left(\begin{array}{l}
x \\
y \\
z \\
t
\end{array}\right)=B_{i j}\left(\begin{array}{c}
x^{\prime} \\
y^{\prime} \\
z^{\prime} \\
t^{\prime}
\end{array}\right) \text { and }\left(\begin{array}{c}
x^{\prime} \\
y^{\prime} \\
z^{\prime} \\
t^{\prime}
\end{array}\right)=A_{i j}\left(\begin{array}{c}
x \\
y \\
z \\
t
\end{array}\right)
$$

Photon contains electromagnetic field accompanying the gravitational field [5-7]. Now, it will be unnecessary to discuss in details of the previous work [3] that electromagnetic field and gravitational field are unified in SSP by the relation (4) which are not applicable in super system. Using the concept of Lorentz transformation in super system we obtain the picture of 
SSP as said above where, $A_{i j}$ and $B_{i j}$ in (11) and (12) are coordinate transformation matrixes in SSP. Now using $A_{i j}$ instead of $Z_{i j}$ and $B_{i j}$ instead of $\bar{Z}_{i j}$ we obtain from (4) the relations between electromagnetic field and gravitational field in SSP

$$
G_{\alpha}^{\prime}\left(r^{\prime}, t^{\prime}\right)=\chi A_{i j} \psi_{\alpha}(r, t), \quad \psi_{\alpha}(r, t)=\bar{\chi} B_{i j} G_{\alpha}^{\prime}\left(r^{\prime}, t^{\prime}\right)
$$

where, $\psi_{\alpha}(r, t)$ and $G_{\alpha}^{\prime}\left(r^{\prime}, t^{\prime}\right)$ are respectively electromagnetic field and gravitational field, $\chi$ and $\bar{\chi}$ are two constants.

Again, an electromagnetic interaction is happening in nucleus due to spin-orbital interaction of proton. Extending the concept of SSP, it is also claimed in [8] that this electromagnetic interaction causes the strong field in nucleus and relation between strong field $\left[\bar{G}_{\alpha}(r, t)\right]$ and electromagnetic field $\left[\psi_{\alpha}^{\prime}\left(r^{\prime}, t^{\prime}\right)\right]$ is

$$
\bar{G}_{\alpha}(r, t)=\bar{\Upsilon} \bar{S}_{i j} \psi_{\alpha}^{\prime}\left(r^{\prime}, t^{\prime}\right)
$$

where, $\bar{S}_{i j}$ is the transformation matrix as discussed in [8] and $\bar{\Upsilon}$ is a constant.

\section{SIGNIFICANCE OF ANGLE BETWEEN DIRECTION OF ANGULAR VELOCITY AND LINEAR VELOCITY OF PHOTON}

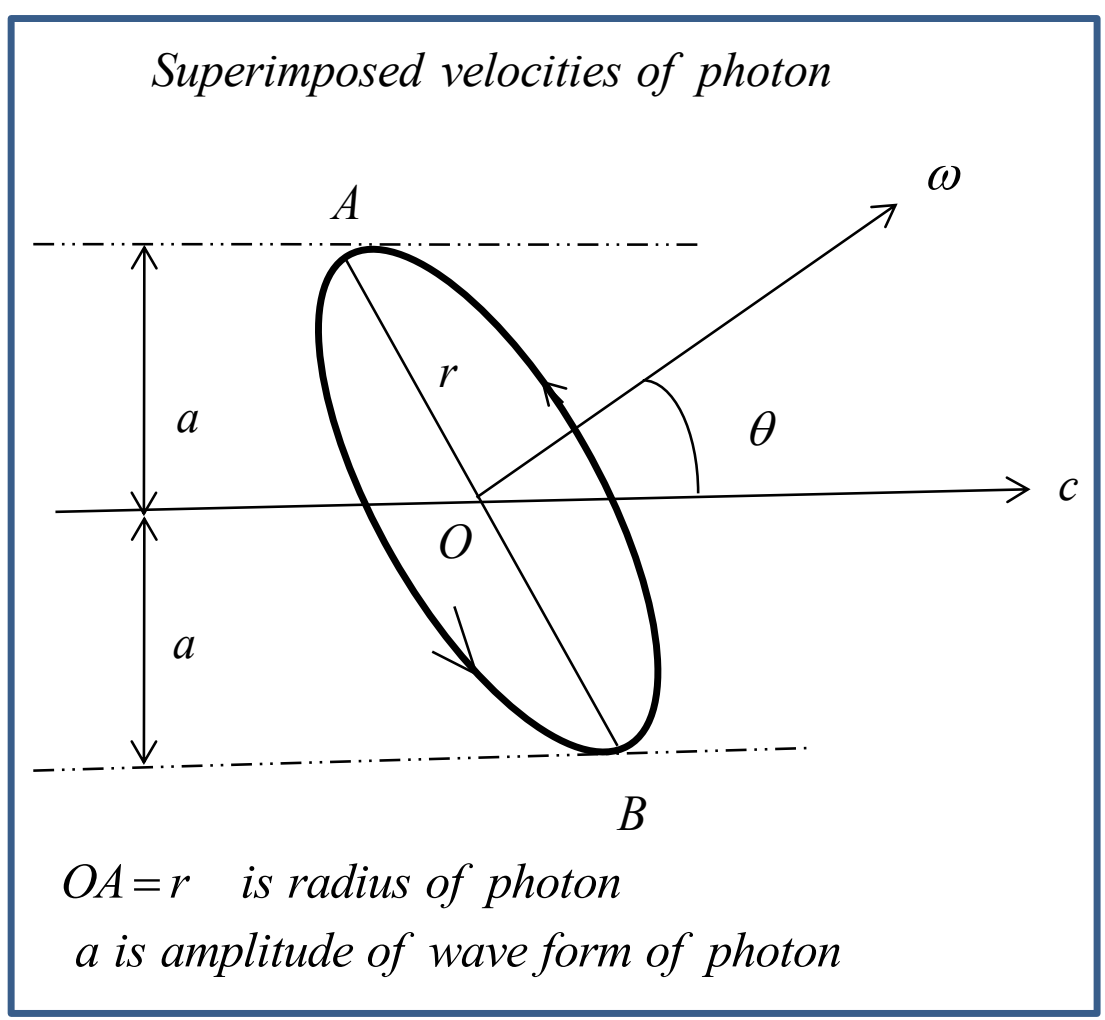

Figure 1. Superimposed velocities of proton. 
Angle $\theta$ in matrices $A_{i j}$ and $B_{i j}$ respectively in (11) and (12) implies that there is an angle between directions of angular velocity $(\omega)$ and linear velocity $(c)$ of photon. Again it is also said that Photon may be considered as a small mass concentrated in a ring of radius $r$ and rotates at velocity of light and it also has linear motion with velocity of light along the axis of rotation. Therefore, if the frequency and wave length of a photon respectively are $v$ and $\lambda$ then $2 \pi r v=c$ and $v \lambda=c$. This leads to the relation $r=\lambda / 2 \pi$.So, amplitude (as shown in the figure-1) of the wave of that photon is

$$
a=r \cos \theta=(\lambda \cos \theta) / 2 \pi
$$

Equation (15) implies that amplitude of the wave form of a photon depends on angle between directions of two velocities of photon as said above.

\section{CONCLUSIONS}

If it be considered that a particle is moving with a velocity same as that of light then, frame of the particle would be a super frame and the system is known as super system. Lorentz transformation in super system makes easy the transformation of co-ordinates from one frame to another frame. Also it removes the contradiction between super system and inertial system.

According to the experimental evidence of Tolmar [5] and others, photon contains electromagnetic field accompanying the gravitational field. Relation between these two fields may be as shown in (14). Again, extending the concept of SSP, relation between strong field and electromagnetic field has been proposed as shown in (15). But these are only a theoretical or mathematical work. So, if we can create a system in which a particle having electric charge possess a spin-spin or spin-orbital interaction like that of proton in nucleus then, electromagnetic field of it may create strong field in nucleus and this will be the proof of the theory.

\section{References}

[1] W.G.V. Rosser, Introductory Special Relativity, Taylor \& Francis, London, 1991,

[2] M. C. Das, R. Misra, "Relativistic Spin of an Arbitrary Body," Advance Studies in Theoretical Physics 6(3) (2012) 135-138.

[3] M.C. Das, R. Misra, A Classical Approach of Unified Field, International Letters of Chemistry, Physics and Astronomy, 7(2) (2013) 73-84.

[4] Y. Rocard, Thermodynamique, Masson, Paris 1957, p. 250.

[5] R. C. Tolmar, Relativity, Thermodynamics and Cosmology, Oxford University Press, Oxford 1934, p. 272. 
[6] D. F. Crawford, Nature 254 (1975) 313-314.

[7] S. C. İnan, Chinese Physics Letter 29 (2012) 031301

[8] M.C. Das, A Prediction in the Way of field Unification, Research Journal of Physical Sciences 1(9) (2013) 1-3. 